SUBJECT AREAS:

SEMICONDUCTORS

NANOPARTICLES

STRUCTURAL PROPERTIES

Received

4 February 2014

Accepted

6 June 2014

Published

7 November 2014

Correspondence and requests for materials should be addressed to L.S. (Ishao@tamu.edu)

\section{Physical assembly of Ag nanocrystals on enclosed surfaces in monocrystalline $\mathrm{Si}$}

\author{
Michael S. Martin' , N. David Theodore' ${ }^{2}$ Chao-Chen Wei ${ }^{3} \&$ Lin Shao $1,2,3$
}

'Department of Nuclear Engineering, Texas A\&M University, College Station, TX77843, ${ }^{2} \mathrm{CHD}-$ Fab, Freescale Semiconductor, Inc., Chandler, AZ 85224, ${ }^{3}$ Materials Science and Engineering Program, Texas A\&M University, College Station, TX 77843.

Growth of thin crystals on external substrate surfaces by many different methods is a well-known technique, but its extension to inner, enclosed surfaces of large defects in monocrystalline materials has not yet been reported. The literature on thin film growth and defects in materials can be leveraged to fabricate new structures for a variety of applications. Here we show a physical process of nucleation and evolution of nanocrystalline silver inside voids in monocrystalline silicon. We found that the Ag growth is

hetero-epitaxial using a coincident site lattice. Alignment of $\mathrm{Ag}$ and $\mathrm{Si}$ atomic planes is uniformly observed by high resolution transmission electron microscopy and macroscopically by channeling Rutherford backscattering spectrometry.

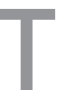
hin films with large lattice mismatch are generally accompanied by several stress-relieving defects due to the large growth area. Growth of such materials inside open-volume defects in crystalline materials has the advantage of perfectly clean, uncontaminated surfaces as well as limited growth volume reducing stressinduced defects, resulting in a physical method to fabricate nanocrystals. The materials $\mathrm{Ag}$ and $\mathrm{Si}$, immiscible below the eutectic point $830^{\circ} \mathrm{C}^{1}$, are chosen because Ag atoms do not form a compound but chemisorb on inner surface of voids ${ }^{2,3}$. The diffusion of $\mathrm{Ag}$ atoms through the Si lattice is slow and is mediated by point defects $\mathrm{s}^{4-6}$, causing localized strains in the $\mathrm{Si}$ lattice which are relieved upon Ag chemisorption inside a void. The lattice mismatch between $\mathrm{Ag}$ and $\mathrm{Si}$ is approximately 25\%, yet hetero-epitaxial growth of $\mathrm{Ag}$ on $\mathrm{Si}(111)$ using a 4:3 coincident site lattice with a small amount of residual strain has been reported ${ }^{7,8}$. Epitaxially oriented $\mathrm{Ag}$ islands have been grown on $\mathrm{H}$-terminated $\mathrm{Si}$ surface with a co-incident site lattice relationship that are strained $\varepsilon=$ $-0.3 \%{ }^{8}$. Previous studies on the efficiency of defects to trap metals in transistors investigated the removal of metal from sensitive regions of micro-electronic devices, with limited focus on promoting metal growth inside defects $^{9-11}$.

Metal nanoparticles can be fabricated in $\mathrm{SiO}_{2}$ by direct implantation of silver ions ${ }^{12}$, however, implantation into silicon causes amorphization ${ }^{13}$ and epitaxial regrowth of silicon is impossible due to high metal concentration ${ }^{14}$. In this study, a low density of large voids is obtained by helium ion irradiation and high temperature defect annealing, which favors growth of voids over dislocations ${ }^{15,16}$. Overcoming the difficulties mentioned would result in a novel composite material of metal nanoparticles fully integrated within a monocrystalline semiconductor substrate suitable for devices or detectors. We seek a structure which (a) forms pure metal nanoparticles instead of metal silicide for maximized electron density, (b) has minimized defects, i.e. dislocations, to avoid leakage currents, and (c) has well-ordered metal-silicon interfaces to minimize carrier recombination.

One potential application of Ag nanocrystals in silicon is for decreasing the thickness of photovoltaic cells due to near- and far-field effects of plasmonic interactions with light ${ }^{17}$. Randomly dispersed nanoparticles ${ }^{18}$ and patterned arrays ${ }^{19-21}$ deposited on the surface of solar cells as well as plasmonic back contacts ${ }^{22}$ have shown moderate to strong effects on induced photocurrent. For nanoparticles with diameters $10-50 \mathrm{~nm}$ interacting with $\mathrm{c}-\mathrm{Si}$, ordinarily forbidden direct transitions across the band-gap are facilitated by momentum of plasmon resonances ${ }^{23,24}$. The resonance frequency of spherical metallic particles in air is in a wavelength region where c-Si photocells readily absorb sunlight, but this resonance can be red-shifted by changing the size, shape and dielectric environment ${ }^{23}$. A shape with a high aspect ratio combined with high refractive index of silicon may red-shift the plasmon resonace near the Si bandgap ${ }^{23}$. The realization of a structure to support surface plasmon polaritons within monocrystalline silicon would significantly expand existing optoelectronic technology.

Figures 1a-d illustrate the major fabrication steps used in our study. First, nanovoids are introduced by low fluence helium ion irradiation which minimizes additional crystalline disorder. Second, annealing is used to relax 


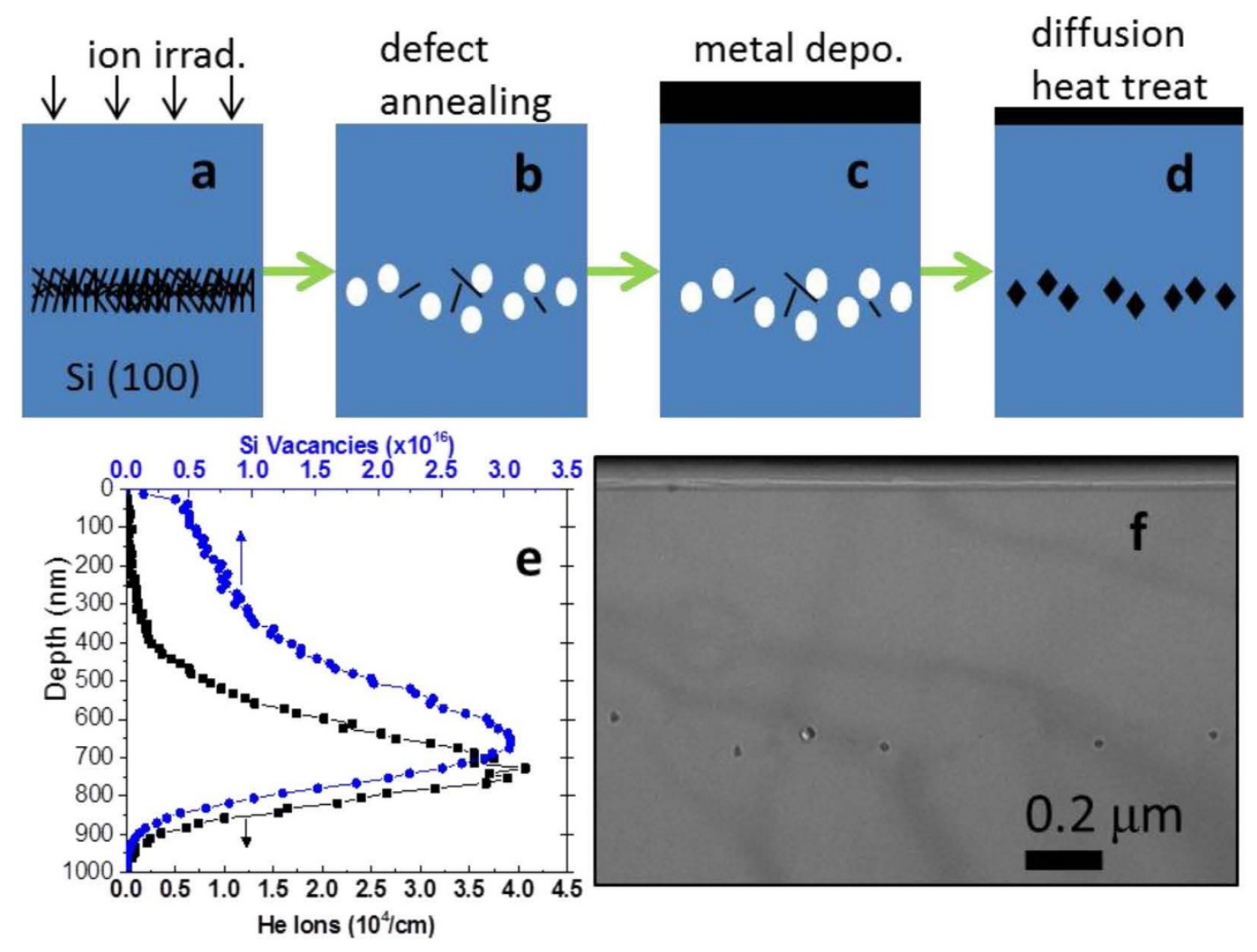

Figure 1 Silver nanoparticles in silicon. Fabricating nanoparticles by (a) ion implantation and (b) defect annealing to create open volume defects, followed by (c) metal deposition and (d) heat treatment to diffuse metal to the open volume defects. (e) Vacancy and He ion concentration profiles calculated by binary collision approximation code SRIM (25). (f) Bright field TEM of Ag nanoparticles in Si.

the voids, so that the initially spherical voids grow and transition into the equilibrium shape for Si crystals, with (111) having lowest surface energy ${ }^{25}$. Third, an immiscible metal is vapor-deposited avoiding adhesion layers containing silicide-forming metals. And finally, metal atoms diffuse from the Si surface by the kick-out or dissociative mechanisms into $\mathrm{Si}$, where open volume defects act as termination sites for metal diffusion. We use a lower temperature for Ag drive-in diffusion to avoid the eutectic temperature of the Ag-Si system $\left(830^{\circ} \mathrm{C}\right)$ but high enough for sufficient diffusivity and epitaxial growth ${ }^{1,4-6}$. Fig. 1e plots the vacancy profile and helium ion distribution, predicted from Monte Carlo simulation code SRIM using full damage cascade model ${ }^{26}$. Due to the low solid solubility of helium in $\mathrm{Si}$, the implanted helium diffuses back to the vacancy peak region to induce and stabilize bubble formation before diffusing out of the $\mathrm{Si}$ wafer, leading to void relaxation ${ }^{16}$. The transmission electron micrograph (TEM) in Fig. 1f shows the structures resulting from $5 \times$ $10^{15} \mathrm{~cm}^{-2} 100 \mathrm{keV} \mathrm{He}$ ion irradiation followed by defect annealing for 60 minutes, evaporation of $100 \mathrm{~nm} \mathrm{Ag} \mathrm{layer} \mathrm{and} \mathrm{diffusion} \mathrm{heat}$ treatment for one hour at $750^{\circ} \mathrm{C}$. The low He ion irradiation fluence is less than or equal to the minimum fluence previously reported to induce voids in silicon ${ }^{27}$. Silver nanoparticles with diameters of up to $\sim 35 \mathrm{~nm}$ are found in a band up to $100 \mathrm{~nm}$ thick. Some of the voids are fully decorated, and the remaining free volume in each void is highly variable.

Different nanoparticle morphologies formed under different annealing and diffusion conditions are compared in Figures $2 \mathrm{a}-\mathrm{d}$. Fig. $2 \mathrm{a}$ and $2 \mathrm{~b}$ correspond to the same defect annealing conditions $\left(950^{\circ} \mathrm{C}\right.$ for 10 minutes) but the Ag diffusion conditions are different ( $\mathbf{a}$ is annealed at $750^{\circ} \mathrm{C}$ for one hour and $\mathbf{b}$ is annealed at $750^{\circ} \mathrm{C}$ for two hours). Fig. $2 \mathrm{c}$ and $2 \mathrm{~d}$ differ in defect annealing time $\left(950^{\circ} \mathrm{C}\right.$ for 30 and 60 minutes, respectively) but share the same diffusion heat treatment $\left(750^{\circ} \mathrm{C}\right.$ for one hour). The same specimen in Fig. $2 \mathrm{~d}$ is shown in Fig. 1f. The areal density of Ag trapped in the void region shown in Fig. 2e is measured by Rutherford backscattering spectrometry (RBS), and is converted to the equivalent thickness of a continuous film with density of bulk $\mathrm{Ag}, 10.47 \mathrm{~g} / \mathrm{cm}^{3}$. Based on the assumption of the bulk density of $\mathrm{Ag}$ which will be justified later, it is clear that a significant amount of metal is transported and trapped in a highly localized volume of the Si wafer. It is expected that increasing the defect annealing time should increase void size, decrease void density, and decrease point defect concentrations, thereby reducing the radiation-enhanced diffusivity of Ag from the Si surface. Furthermore, it is expected that increasing the diffusion heat treatment time should promote Ag growth inside voids. However, metastable void decoration is observed in $2 \mathbf{a}$ and $2 \mathbf{b}$, which contain voids of roughly equivalent size. Silver atoms are trapped, or chemisorbed, in voids after a short time but re-emitted into silicon upon increasing heat treatment. RBS analysis shown in 2e supports this observation, and it is well known that void gettering of $\mathrm{Au}$ in $\mathrm{Si}$ is metastable $^{3}$. The dramatically increased Ag trapping as the defect annealing time increases from 30 to 60 minutes, shown in $2 \mathrm{c}$, d, and $\mathrm{e}$, is unexpected due to the requirement of $\mathrm{Ag}$ to diffuse with the aid of Si point defects. Lower point defect concentrations caused by increased defect annealing lowers Ag diffusivity, which should lead to decreased Ag trapping inside voids. The opposite trend is measured macroscopically by RBS and observed microscopically by TEM. Voids are larger and dislocations (indicated by strain contrast) are fewer after longer defect annealing. Larger voids, fewer other defects (dislocations), and relatively suppressed diffusivity (though still enhanced over pristine Si) contribute to higher Ag trapping in voids in Fig. $2 \mathrm{c}$ and $2 \mathrm{~d}$.

Figure $3 \mathrm{a}$ and $\mathrm{b}$ shows high resolution TEM and Fourier filtering of one nanoparticle viewed along the [110] zone axis in slightly different focusing conditions. This specimen has also been shown 

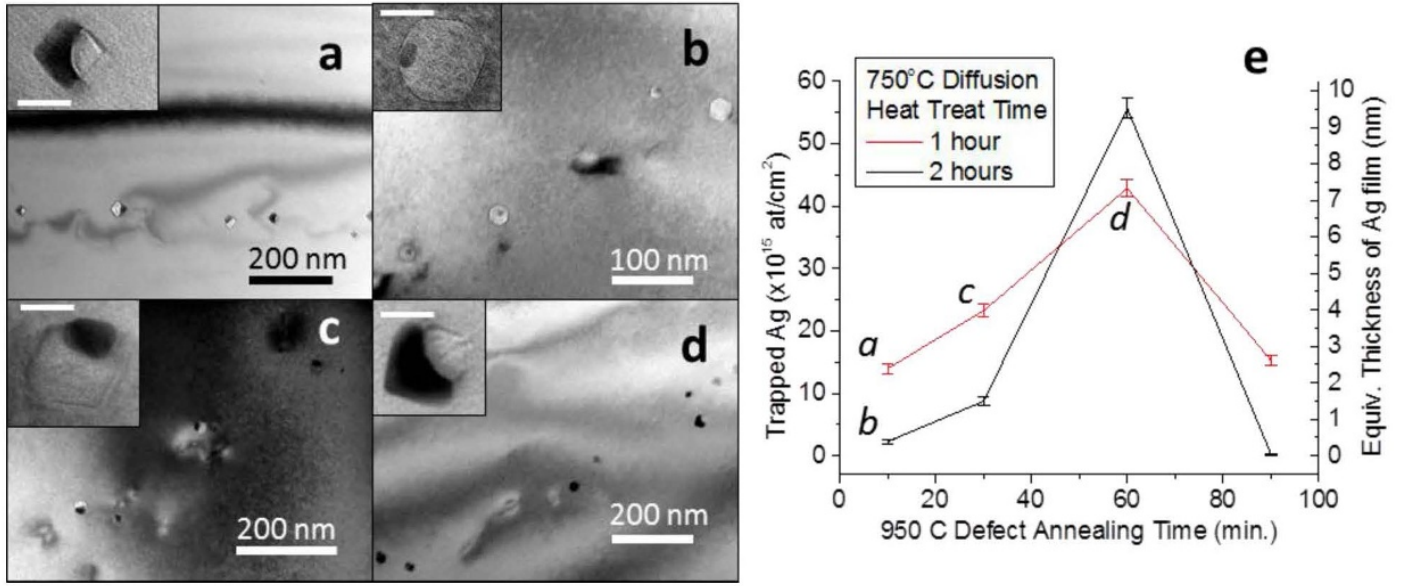

Figure $2 \mid$ Microscopic and macroscopic quantification of Ag trapped in voids. Annealing at $950^{\circ} \mathrm{C}$ for $10 \mathrm{~min}(\mathrm{a} \& \mathrm{~b}), 30 \mathrm{~min}(\mathrm{c})$, and $60 \mathrm{~min}$ (d) forms voids. Heat treatment at $750^{\circ} \mathrm{C}$ for one hour (a, c, \& d) and two hours (b) diffuse Ag from surface to voids. Scale bars in insets of (a-d) are equivalent and represent $20 \mathrm{~nm}$. (e) Amount of Ag trapped in nanoparticle region is measured by Rutherford backscattering spectrometry (RBS), with points labeled $a-d$ corresponding to TEM. The right abscissa shows equivalent thickness of a continuous Ag film.

in Figs. If and 2d. The electron beam is in a double diffraction alignment condition, where electrons are diffracted by multiple atomic planes. In the double diffraction condition, Moire interference is caused by interaction of the electron beam with layers of parallel $\mathrm{Ag}$ and $\mathrm{Si}$ atomic planes which have different spacings. The fringe spacings of the dashed lines in Fig. $3 \mathrm{a}$ and $3 \mathrm{~b}$ are $5.5 \AA$ and $9.4 \AA$, respectively. The interference fringe spacing for parallel $\mathrm{Si}(111)$ and $\mathrm{Ag}(111)$ planes is calculated by

$$
L_{111}=\frac{d_{S i(111)} d_{A g(111)}}{d_{S i(111)}-d_{A g(111)}}=\frac{(3.135 \AA)(2.358 \AA)}{3.135 \AA-2.358 \AA}=0.951 \AA
$$

where $d_{A(x y z)}$ is the spacing of plane $(x y z)$ of element $\mathrm{A}^{28}$. For $\mathrm{Si}(110)$ and $\operatorname{Ag}(110)$ planes with spacings of $1.919 \AA$ and $1.444 \AA$, respectively, Eq. 1 gives interference fringe spacing of $5.6 \AA$. The close agreement between measured and calculated values and the shared orientation of interference fringes through nanoparticles with Si lattice planes outside nanoparticles supports the inference that atomic planes of $\mathrm{Ag}$ in the nanocrystals are parallel to Si substrate lattice planes.

Detailed TEM investigation of two nanocrystals imaged along the [110] zone axis is shown in Fig. 4 from the specimen shown in Fig. $2 c$ and point labelled "c" in Fig. 2e. Two nanocrystals shown in Fig. 4a are approximately the same size, but the voids they are decorating clearly have different sizes. The kinetics of nanocrystal growth depend on the diffusion conditions (time, temperature, point defect concentrations) rather than the initial void size. The nanocrystal decorating the smaller void on left in Fig. 4a is analyzed by highresolution TEM in Fig. 4b, and lattice directions determined from the

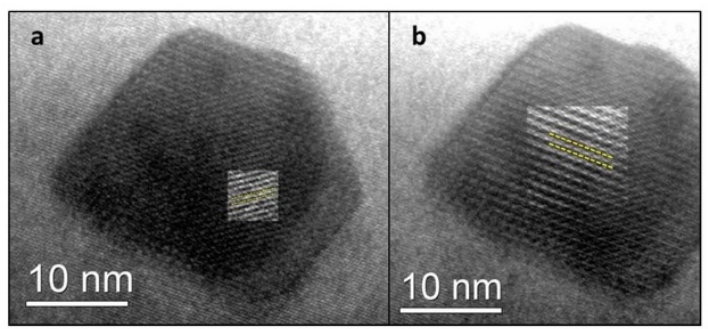

Figure 3 Fourier filtered high resolution TEMs of one Ag nanoparticle taken from specimen shown in Fig. 2d showing Moiré interference of Ag and Si (a) (110) atomic planes with fringe spacing $5.5 \AA$ and (b) (111) atomic planes with fringe spacing $9.4 \AA$. Dashed lines indicate fringes. corresponding diffraction pattern (not shown) are indicated. Moiré interference with a fringe spacing of $9.9 \AA$, indicated by grey dashed lines, is readily visible without the aid of a Fourier filtering method. The nanocrystal in Fig. $4 \mathrm{~b}$ is bounded primarily by (111) atomic planes and a smaller area of (100) atomic planes. This corresponds well with the surface energy anisotropy reported for pure $\mathrm{Si}$, where the lowest energy surface is (111) and the next lowest is $(100)^{25}$. The shape is a rounded octahedron. No stress fields, which would indicate large structural distortion, at the $\mathrm{Si} / \mathrm{Ag}$ interface are observed. However, the cross-sectional TEM sample thickness is much greater than the nanoparticle size, and so small distortions may be averaged out by the large amount of $\mathrm{Si}$ above and below the nanocrystal.

Changes to the Si lattice caused by diffusion and trapping of $\mathrm{Ag}$ is quantified by channeling ion beam analysis of the $<100>$ (surfacenormal) axis. Channeling and random spectra obtained with $2 \mathrm{MeV}$ helium analysis beam around the $\mathrm{Si}<100>$ axial channel direction are shown in Figure 5a, obtained from a sample with $950^{\circ} \mathrm{C}$ defect annealing for 30 minutes and $750^{\circ} \mathrm{C}$ diffusion heat treatment for one hour (characterized by TEM in Figs. $2 \mathrm{c}$ and 4 ). The spectrum for channel numbers $<290$ corresponds to backscattering from $\mathrm{Si}$, while that for channel numbers $>290$ corresponds to backscattering from $\mathrm{Ag}$ (yields are enlarged by a factor of 20 to aid visualization). The channeling "Si + Ag NP" surface peak (channel 265) shows that the Si surface has not reconstructed or formed a compound layer when compared with the pure Si channeling surface peak. The sharp peaks close to channel 330 in the random spectrum (345 in the channeling spectrum due to $\mathrm{He}$ ion stopping power difference)

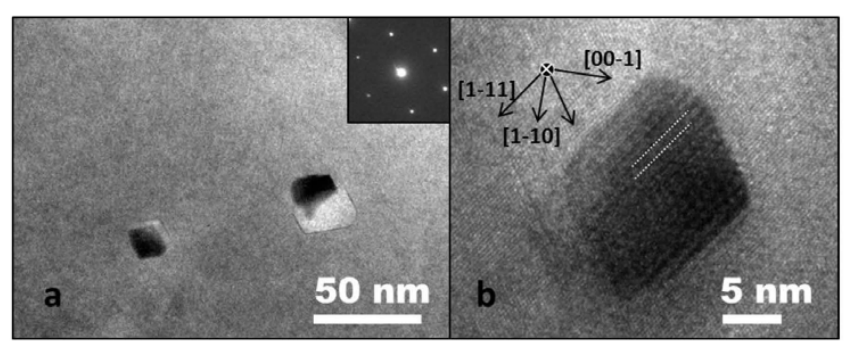

Figure $4 \mid$ High resolution TEM of Ag nanocrystals with free volume taken from specimen shown in Fig. 2c. (a) Two nanocrystals of equivalent size grown in voids of different size and (b) left nanocrystal in (a) imaged along [110] zone axis showing Moiré interference of $\mathrm{Ag} / \mathrm{Si}$ (111) atomic planes with fringe spacing $9.9 \AA$ visible without Fourier filtering of micrograph. 
correspond to Ag nanocrystals. RBS spectra with channel number less than 280 correspond to backscattering from Si. At the channel number 200 and below, the RBS channeling spectrum (blue curve) has a bump-like yield enhancement, instead of a peak. This means that the defects in the Ag nanocrystal/void region are strong dechanneling centers like dislocations, instead of direct backscattering centers like defect clusters. On the other hand, TEM in Fig. $2 c$ shows that the Ag NP region is relatively defect free. Figure $5 \mathrm{~b}$ compares channeling RBS spectra of Ag in Si samples obtained under different thermal treatments to an unaltered Si wafer. The region between the Si surface and the voids/nanocrystals is shown in channels $>$ 200 , and the void/nanocrystal region starts around channel 200 extending deeper into the wafer. The slightly increased backscattering yield between channels 200-260 of 60 minutes vs. 30 minutes (of defect annealing at $950^{\circ} \mathrm{C}$, diffusion heat treatment for one hour at $750^{\circ} \mathrm{C}$ ) indicates that the Si point defect concentration does not monotonically decrease with increasing defect annealing. Based on this observation, the increased Ag trapping observed in Fig. $2 \mathrm{c}$ and $2 \mathrm{~d}$ is caused by greater Ag diffusion mediated by greater point defect concentrations. At channel number 200 and lower, yields increase with decreasing channel numbers. Except for the condition involving the annealing at $750^{\circ} \mathrm{C} / 90 \mathrm{~min}$, yields after the "bump" region (channel 180 to 200) do not decrease, suggesting the existence of strong dechanneling centers in each sample. For the annealing at $750^{\circ} \mathrm{C}$ for $90 \mathrm{~min}$, the yield is reduced after the Ag nanocrystal region and becomes close to that of unaltered Si. A small amount of $\mathrm{Ag}$ remaining in this sample is in the form of small defect clusters instead of nanocrystals. Empty voids, from which all or most Ag has been reemitted into the Si lattice, also cannot confine the He beam and would scatter the ion beam similar to point defects. As the beam exits the void and re-enters the Si substrate, the void/substrate interface causes direct scattering (similar to the phenomenon causing the surface peak typically observed in channeling curves). This argument is supported by TEM in Fig. 2. Although dislocations are observed under TEM, their densities are too low to contribute to such strong dechanneling in the rest of the samples analyzed in Fig. 5b. We argue that the dechanneling is caused by the Si stress and slight atomic plane bending near the Ag nanocrystals, due to mismatch in the number of atomic planes of $\mathrm{Ag}$ and $\mathrm{Si}$ at the interface creating a small amount of residual strain ${ }^{8}$. We believe that the dechanneling is caused by $\mathrm{Si}$ structural distortion near the $\mathrm{Ag}$ nanocrystals. Interaction with $\mathrm{Ag}$ atomic planes that dechannel the analysis beam resulting in backscattering from nearby $\mathrm{Si}$ can also happen, however the geometric cross section of Ag nanocrystals is too small to account for the observed dechanneling cross section. Epitaxial growth through $4: 3$ co-incident site lattice (CSL) leads to localized stress fields extending into the surrounding $\mathrm{Si}$, which bend the He ion beam and causes dechanneling in a way similar to dislocations. Furthermore, the absence of defect peaks (direct scattering rather than dechanneling) suggests that there is no Si disorder similar to point defects or small defect clusters at the Si-Ag interface. We can extract the depth profile of dechanneling centers by adopting the equation widely used for dislocations

$$
\lambda n_{d}=-\frac{d}{d t}\left[\ln \left(\frac{1-\chi_{d}(t)}{1-\chi_{v}(t)}\right)\right]
$$

where $\chi_{d}(t)$ is the normalized channeling yield from channel $t$ in each sample and $\chi_{v}(t)$ is the normalized channeling yield in channel $t$ from pure, unadulterated $\mathrm{Si}^{29} . \lambda$ is the dechanneling cross section which depends on the size of the region effected by the stress fields from the $\mathrm{Si}$-Ag interfaces and $n_{d}$ is the concentration of dechanneling centers. The measured defects are an overestimate of the true number of carrier recombination centers.

Angular scans across the surface-normal $<100\rangle$ and off-normal $<110>$ axial channels are shown in Figs. $6 \mathrm{a}$ and $6 \mathrm{~b}$, respectively. In Fig. 6b, the ion beam is aligned with the (100) plane channel as it goes
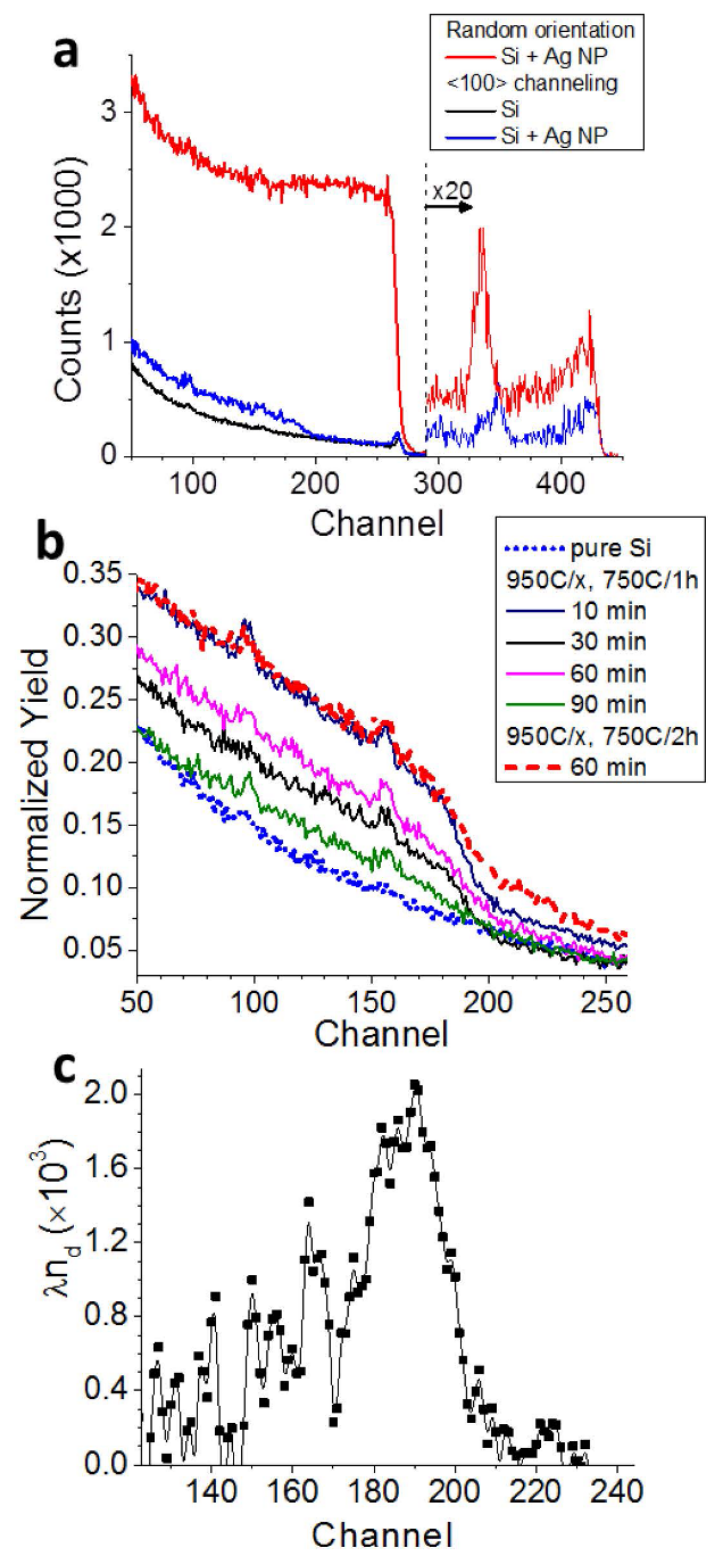

Figure $5 \mid$ Ion channeling analysis obtained from $<100>$ axis of $\mathrm{Ag}$ in $\mathrm{Si}$, (a) RBS channeling of surface normal $<100\rangle$ axial channel with backscattering yield from Ag atoms highlighted. Comparison with $<100>$ axial channeling of pure $\mathrm{Si}$ is included. (b) Normalized backscattering yield of Ag in Si samples fabricated with defect annealing times of 10, 30, 60, and 90 minutes and diffusion heat treatment times of one or two hours. (c) Dechanneling cross section of Si extracted from (a).

through the $<110>$ axial channel, causing normalized yield in the "shoulders" to be less than unity. The yields from Si near the surface (without Ag) are plotted for comparison. The yields are integrated from Ag nanoparticle regions, and normalized to their corresponding random values. All yield curves show a dip at zero tilt with respect to the axial channel position, which means that the Ag atomic rows are aligned with those of $\mathrm{Si}$ in both $<100>$ and $<110>$ directions, suggesting epitaxial growth of Ag. Under CSL configuration, a fraction of the aligned Ag atomic rows are not aligned with the Si atomic rows and are viewed as dislocations by the channeled beam. This increases the Ag yield and causes higher Ag minimum yields when compared with $\mathrm{Si}$, so the physical meaning of the observed minimum yield of Ag nanocrystals is not known. 


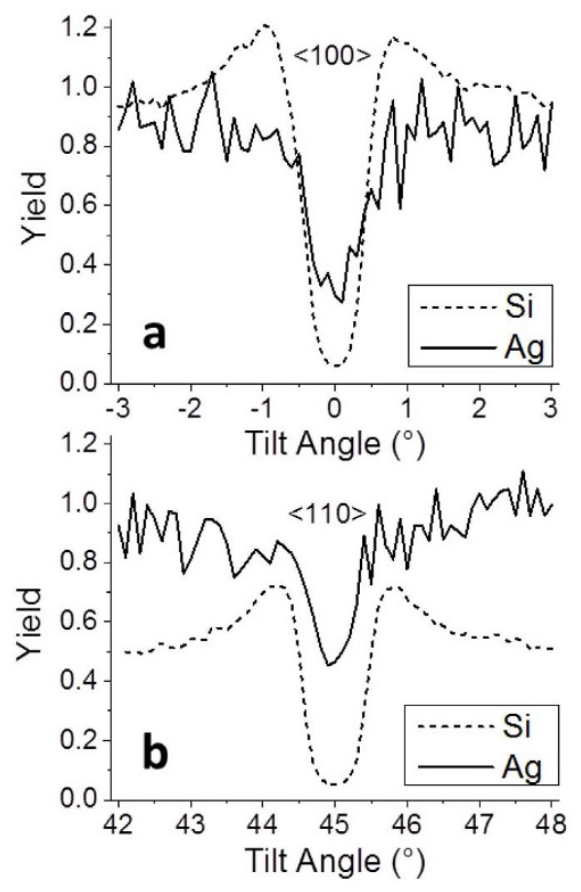

Figure 6 Ion channeling of $\mathrm{Ag}$ in Si fabricated with $950^{\circ} \mathrm{C}$ defect annealing for 30 minutes and $750^{\circ} \mathrm{C}$ diffusion heat treatment for one hour. Angular scans across the $<100\rangle$ (surface normal) axis (a) and the $<110>$ (off-normal) axis aligned with (100) plane (b).

For device applications, the Schottky barrier height of the Ag-Si interface is around $0.6 \mathrm{eV}$, which suggests a threshold photon wavelength of $\sim 1.8 \mu \mathrm{m}$ for photoemission from Ag particles (28), and the presence of an ideal interface could decrease that value ${ }^{30,31}$. Therefore, in addition to use as a computing component driven by $1.3 \mu \mathrm{m}$ or even $1.55 \mu \mathrm{m}$ semiconductor lasers, devices can be used for radiation detection that require high spatial resolution since the substrate is compatible with existing Si technology. It is possible to extend applications further into the infrared region, for imaging applications, through strain engineering to narrow the band gap of the semiconductor medium, reducing the Schottky barrier height. For example, the band gap of a $\mathrm{Si}_{0.7} \mathrm{Ge}_{0.3}$ layer grown in a relaxed $\mathrm{Si}_{0.3} \mathrm{Ge}_{0.7}$ structure can be reduced to $0.8 \mathrm{eV}$, and thus the Schottky barrier height can be reduced to $0.2 \mathrm{eV}$ for a $\mathrm{Ag}-\mathrm{Si}$ interface ${ }^{32}$. Consequently, the threshold wavelength for device switching would be increased to $6.2 \mu \mathrm{m}$.

The present study does not involve annealing at temperatures greater than the eutectic point of $\mathrm{Si}$ and $\mathrm{Ag}$, therefore mixing is not expected. Previous reports have suggested metastable silver silicide phases created under specialized conditions such as rapid quenching due to melt spinning or high pressure causing phase transformation from cubic to tetragonal Si unit cell in small volume effected by nanoindentation ${ }^{33,34}$. However, our experimental conditions do not involve rapid cooling and voids are not pressurized shown by transition to faceted from spherical shape. Very limited solid solubility of $\mathrm{Si}$ and $\mathrm{Ag}$ in each other leads to high likelihood of heterogeneous epitaxial growth. The occurrence of Moire interference fringes parallel to $\mathrm{Si}$ atomic planes with spacings near calculated values provides strong evidence of pure Ag nanocrystals. The nanocrystals are too small (and surrounding Si too thick) to make easy identification of phases from electron diffraction patterns of lowindex zone axes possible in this case. Supplementary Figures S3 and $\mathrm{S} 4$ contains TEM and electron diffraction patterns from a much larger Ag nanocrystal that was fabricated apart from this study which clearly demonstrates pure $\mathrm{Si}$ and $\mathrm{Ag}$ phases.
The synthesized structure realizes, in a new way, the plasmonic structure to improve photovoltaic devices proposed by Atwater and Polman ${ }^{17}$. Fully integrating nanometallic plasmonics with dielectric photonics is now possible ${ }^{35}$. Development of plasmonic theory for non-spherical metallic nanoparticles must be carried out before applications will be well understood. This physical method of growing nanocrystals of an immiscible material on the inner surface of open volume defects in a crystalline solid has potential to further tune the properties of technologically important materials in ways that have not been possible previously.

\section{Methods}

Fabrication of Ag nanoparticles in voids in monocrystalline silicon. The method to fabricate nanoparticles by chemisorption onto an inner surface consists of three steps: create open volume defects in Si, deposit Ag on the Si surface, and diffuse Ag into the open volume defects. Voids are created by two steps, first implanting $5 \times 10^{15}$ $100 \mathrm{keV} \mathrm{He}$ ions $/ \mathrm{cm}^{2}$ at room temperature into float-zone, B-doped $\mathrm{Si}(100)$ wafer of resistivity 5-10 $\Omega-\mathrm{cm}$. Defect annealing to nucleate and grow voids is performed at $950^{\circ} \mathrm{C}$ in flowing ultra-high purity Ar for 10, 30, 60 or 90 minutes utilizing a hot-zone method. The sample rests in a quartz boat and is inserted into and withdrawn from the hot zone of furnace as rapidly as possible. After defect annealing and before physical vapor deposition (PVD), sample surfaces are cleaned by sequential acetone and ethanol baths followed by evaporation with dry nitrogen gas, performed twice, then etching with $\mathrm{HF}$ acid vapor for $10 \mathrm{~s}$. Evaporation of $100 \mathrm{~nm} \mathrm{Ag}$ onto the $\mathrm{Si}$ surface is performed in a BOC Edwards Auto 306 Metal Evaporation Chamber. No adhesion layer (e.g. Cr) is deposited. Heat treatment to diffuse Ag into the Si wafer is performed at $750^{\circ} \mathrm{C}$ in ultra-high purity Ar utilizing a hot-zone method. Prior to characterization, residual Ag is removed by wetting the surface with solvent (e.g. acetone) and wiping with Kimwipes or cotton swabs.

Microscopic characterization. Cross-sectional samples for transmission electron microscopy are created by the lift-out technique using dual-beam SEM/FIB. TEM specimens are characterized in a Philips CM200 transmission electron microscope operated at $200 \mathrm{kV}$. The spacings of $\mathrm{Ag}$ atomic planes measured from diffraction patterns obtained from the particles are normalized to $\mathrm{Si}$ atomic planes from the matrix. A portion of the original micrograph is Fourier transformed, masked to show Moiré interference fringe and inverted. Fourier filtering is carried out by averaging the portion of the original micrograph with the inverted Fourier transformed image.

Ion beam analysis. Ion beam analysis is performed with a $2 \mathrm{MeV} \mathrm{He}$ ion analyzing beam probing the $\langle 100\rangle$ and $<110\rangle$ axial channels, and the beam is aligned with the (100) plane channel as it scans across the $<110>$ axial channel. A surface barrier detector collects He ions backscattered $165^{\circ}$ from the incident direction.

1. Olesinski, R. W. \& Abbaschian, G. J. Ag-Si (Silver-Silicon) Binary Alloy Phase Diagrams, II Ed. (ASM International, Materials Park, Ohio, 1990).

2. Myers, S. M., Seibt, M. \& Schroter, W. Mechanisms of transition-metal gettering in silicon. J. Appl. Phys. 88, 3795 (2000).

3. Myers, S. M. \& Petersen, G. A. Transport and reaction of Au in Si containing cavities. Phys. Rev. B 57, 7015 (1998).

4. Rollert, F., Stolwijk, N. A. \& Mehrer, H. Solubility, diffusion and thermodynamic properties of silver in silicon. J. Phys. D: Appl. Phys. 20, 1148 (1987).

5. Nason, T. C., Yang, G. R., Park, K. H. \& Lu, T. M. Study of silver diffusion into $\mathrm{Si}(111)$ and $\mathrm{SiO} 2$ at moderate temperatures. J. Appl. Phys. 70, 1392 (1991).

6. Chen, L., Zeng, Y., Nyugen, P. \& Alford, T. L. Silver diffusion and defect formation in $\mathrm{Si}(111)$ substrate at elevated temperature. Mater. Chem. Phys. 76, 224 (2002).

7. Legoues, F. K., Liehr, M., Renier, M. \& Krakow, W. Microstructure of epitaxial Ag/ $\mathrm{Si}(111)$ and $\mathrm{Ag} / \mathrm{Si}(100)$ interfaces. Phil. B 57, 179 (1988).

8. Li, B. Q. \& Zuo, J.-M. The development of epitaxy of nanoclusters on latticemismatched substrates: Ag on H-Si(111) surfaces. Surf. Sci. 520, 7 (2002).

9. Wong-Leung, J., Williams, J. S. \& Nygren, E. Diffusion and trapping of Au to cavities induced by H-implantation in Si. Nucl. Inst. Meth. B 106, 424 (1995).

10. Venezia, V. C., Eaglesham, D. J., Haynes, T. E., Agarwal, A., Jacobson, D. C., Grossmann, H.-J. \& Baumann, F. H. Depth profiling of vacancy clusters in MeVimplanted Si using Au labeling. Appl. Phys. Lett. 73, 2980 (1998).

11. Kinomura, A., Williams, J. S., Wong-Leung, J. \& Petravic, M. Microstructural differences between platinum and silver trapped in hydrogen induced cavities in silicon. Appl. Phys. Lett. 72, 2713 (1998).

12. Fortuna, F. et al. Selective nucleation induced by defect nanostructures: a way to control cobalt disilicide precipitation during ion implantation. J. Appl. Phys. 112, 123504 (2012).

13. Meldrum, A., Honda, S., White, C. W., Zuhr, R. A. \& Boatner, L. A. Nanocrystals in crystalline silicon: Void formation and hollow particles. J. Mater. Res. 16, 2670 (2001).

14. Rimini, E. Ion Implantation: Basics to Device Fabrication (Kluwer, Boston, 1995).

15. Fichtner, P. F. P. et al. Overpressurized bubbles versus voids formed in helium implanted and annealed silicon. Appl. Phys. Lett. 70, 732 (1997). 
16. Corni, F. et al. Helium-implanted silicon: A study of bubble precursors. J. Appl. Phys. 85, 1401 (1999).

17. Atwater, H. A. \& Polman, A. Plasmonics for improved photovoltaic devices. Nature Materials 9, 205 (2010).

18. Schaadt, D. M., Feng, B. \& Yu, E. T. Enhanced semiconductor optical absorption via surface plasmon excitation in metal nanoparticles. Appl. Phys. Lett. 86, 063106 (2005).

19. Hagglund, C., Zach, M., Petersson, G. \& Kasemo, B. Electromagnetic coupling of light into a solar cell by nanodisk plasmons. Appl. Phys. Lett. 92, 053110 (2008).

20. Pala, R. A. et al. Design of plasmonic thin-film solar cells with broadband absorption enhancements. Adv. Mat. 21, 3504 (2009).

21. Munday, J. N. \& Atwater, H. A. Large integrated absorption enhancement in plasmonic solar cells combining metal gratings and antireflection coatings. Nano Lett. 11, 2195 (2010).

22. Ferry, V. E. et al. Light trapping in ultrathin plasmonic solar cells. Opt. Expr. 18, A237 (2010).

23. Kirkengen, M., Bergli, J. \& Galperin, Y. M. Direct generation of charge carriers in c-Si solar cells due to embedded nanoparticles. J. Appl. Phys. 102, 093713 (2007).

24. Jacak, W., Henrykowski, A. \& Marszalski, K. Plasmon enhanced photovoltaic effect in metallically nanomodified photocells. Sol. Energy Mat. Sol. Cells 117, 663 (2013).

25. Eaglesham, D. J., White, A. E., Feldman, L. C., Moriya, N. \& Jacobson, D. C. Equilibrium shape of Si. Phys. Rev. Lett. 70, 1643 (1993).

26. Ziegler, J. F., Ziegler, M. D. \& Biersack, J. P. SRIM-The stopping and range of ions in matter (2010). Nucl. Inst. Meth. B 268, 1818 (2010).

27. Raineri, V., Battaglia, A. \& Rimini, E. Gettering of metals by He induced voids in silicon. Nucl. Inst. Meth. Phys. B 96, 249 (1995)

28. Hirsch, P. B., Howie, A., Nicholson, R. B., Pashley, D. W. \&Whelan, M. J. Electron Microscopy of Thin Crystals (Plenum Press, New York, 1967).

29. Feldman, L. C., Mayer, J. W. \& Picraux, S. T. Materials Analysis by Ion Channeling: Submicron Crystallography (Academic Press, New York, 1982)

30. Tung, R. T. Schottky-Barrier Formation at Single-Crystal Metal-Semiconductor Interfaces. Phys. Rev. Lett. 52, 641 (1984).

31. Weitering, $\mathrm{H}$. H. et al. Inhomogeneous Schottky barrier at $\mathrm{Ag} / \mathrm{Si}(111)$ and $\mathrm{Ag} /$ Si(100) interfaces. J. Appl. Phys. 79, 7820 (1996).

32. Moontragoon, P., Ikonić, Z. \& Harrison, P. Band structure calculations of Si-GeSn alloys; achieving direct band gap materials. Semicond. Sci. Technol. 22, 742 (2007)
33. Olesinski, R. W., Gokhale, A. B. \& Abbaschian, G. J. The Ag-Si (Silver-Silicon) system. Bull. Alloy Phase Diag. 10, 635 (1989).

34. Lee, W.-S., Chen, T.-H., Lin, C.-F. \& Wu, C.-L. Microstructual evolution of nanoindented $\mathrm{Ag} / \mathrm{Si}$ thin-film under different annealing conditions. Mat. Trans. 52, 1868 (2011)

35. Brongersma, M. L. \& Shalaev, V. M. The case for plasmonics. Science 328, 440 (2010)

\section{Acknowledgments}

This work is funded by Welch Foundation project A-1671. M.S.M. and L.S. would like to acknowledge Dr. Wei-Kan Chu and Xuemei Wang of the Texas Center for Superconductivity at the University of Houston for RBS measurements.

\section{Author contributions}

L.S. designed experiments. L.S. and M.S.M. prepared the manuscript. M.S.M. carried out the irradiation experiments. N.D.T. fabricated TEM samples. N.D.T. and C.-C.W.

characterized TEM samples. M.S.M., N.D.T. and C.-C.W. performed TEM data analysis.

\section{Additional information}

Supplementary information accompanies this paper at http://www.nature.com/ scientificreports

Competing financial interests: The authors declare no competing financial interests. How to cite this article: Martin, M.S., Theodore, N.D., Wei, C.-C. \& Shao, L. Physical assembly of Ag nanocrystals on enclosed surfaces in monocrystalline Si. Sci. Rep. 4, 6744; DOI:10.1038/srep06744 (2014).

This work is licensed under a Creative Commons Attribution-NonCommercialShareAlike 4.0 International License. The images or other third party material in this article are included in the article's Creative Commons license, unless indicated otherwise in the credit line; if the material is not included under the Creative Commons license, users will need to obtain permission from the license holder in order to reproduce the material. To view a copy of this license, visit http:// creativecommons.org/licenses/by-nc-sa/4.0/ 